\title{
3D Printed Models Offer Superior Advantages in Medical Student Education
}

\author{
Zhonghua Sun* \\ Professor, Discipline of Medical Radiation Sciences, School of Molecular and Life Sciences, Curtin University, Australia
}

*Corresponding author: Sun Z, Professor, Discipline of Medical Radiation Sciences, School of Molecular and Life Sciences, Curtin University, Australia, Tel: +61-8-9266 7509; Fax: +61-8-9266 2377; E-mail: z.sun@curtin.edu.au

Received: 26 Oct, 2019 | Accepted: 28 Oct, 2019 | Published: 04 Nov, 2019

Citation: Sun Z (2019) 3D Printed Models Offer Superior Advantages in Medical Student Education. J Hear Health 5(2): dx.doi.org/10.16966/2379769X.152

Copyright: ( 2019 Sun Z. This is an open-access article distributed under the terms of the Creative Commons Attribution License, which permits unrestricted use, distribution, and reproduction in any medium, provided the original author and source are credited.

Three-Dimensional (3D) printing has been increasingly used in medical applications ranging from the original orthopaedic and maxillofacial applications to the emerging areas including tumour and cardiovascular disease [1-10]. Use of patient-specific 3D printed models has been shown to play an important role in cardiovascular disease by replicating anatomical structures and pathologies with high accuracy [9-11]. This is especially useful in the domain of Congenital Heart Disease (CHD) due to complexity of cardiac structures and heterogeneity of heart pathologies which are difficult to be appreciated on traditional image visualizations on a $2 \mathrm{D}$ flat screen [10]. 3D printed heart models created from patient's Computed Tomography (CT) or Magnetic Resonance Imaging (MRI) imaging data offer realistic physical models with tactile experience, thus showing great promise in improving understanding of the complex CHD situations (Figure 1).

One of the main applications of 3D printed models in CHD lies in medical education as reported by a recent systematic review and meta-analysis [12]. While most of the other applications of 3D printed models in CHD are dominated by case reports or case series, there are three Randomized Controlled Trials (RCT) available in the literature reporting the usefulness of $3 \mathrm{D}$ printed models in medical education of CHD [13-15]. This is enhanced by a recent study showing further evidence of $3 \mathrm{D}$ printed heart models in medical student education through use of a range of CHD lesions [16].

Smerling J, et al. in their study looked at the 3D printed models in CHD from different perspectives regarding medical education [16]. They selected 6 CHD cases consisting of various cardiac pathologies: pulmonic stenosis, Atrial Septal Defect (ASD), Tetralogy of Fallot (ToF), aortic coarctation, hypoplastic left heart syndrome and d-transposition of the great arteries. 3D physical models of these cardiac pathologies were printed for use in the CHD workshop. Fortyfive first year medical students were invited and randomly assigned to participate in the CHD workshop consisting of four stations on teaching cardiac pathologies: watching an embryology video, 2D diagram with spoken explanation, examining pathology specimens and $3 \mathrm{D}$ printed heart models. Each group was given 15 minutes at each station before proceeding to the next one. When students arrived at the $3 \mathrm{D}$ printed model station, they were asked to complete a pre-and post-intervention survey with the aim of assessing their knowledge and understanding of cardiac lesions, as well as their opinion towards the educational benefit of each modality (presented at each station). Results showed that student's knowledge was significantly improved for every cardiac lesion $(\mathrm{p}<0.001)$ after the use of $3 \mathrm{D}$ printed models. There was a strong positive correlation between the mean knowledge increase and improved understanding of the complexity of $\mathrm{CHD}$ $(\mathrm{p}=0.03)$. Regarding the preferred teaching tools in CHD, pathology, $3 \mathrm{D}$ printed models and spoken explanation were ranked as the most helpful teaching methods with $80.7 \%, 74.2 \%$ and $65 \%$ of students rating them as the top 3 choices, respectively. Further, students strongly agreed that $3 \mathrm{D}$ printed models increased their confidence in explaining and understanding congenital heart anatomy and disease when compared to other modalities (mean score: $4.24 \pm 0.69$ out of 5 ).

There are three findings in this study that bear discussion. First, this study offers insights into how 3D printed models can be used as a valuable alternative approach to current teaching tools for medical students, especially in the area of complex cardiac anatomy and pathology. Currently, there are 4 RCT studies available in the literature with 3 of them focusing on CHD education [13-15], and the remaining one on medical student education in normal cardiac anatomy [17]. Of these 3 studies, two of them showed that 3D printed models improved paediatric resident's knowledge and understanding of CHD [13,14] (Figure 2), while the study by Su W, et al. reported the significant improvement in structural conceptualization in the student group with 3D printed heart models [15]. Smerling J, et al. in their study further validated these findings of the superior advantages offered by $3 \mathrm{D}$ printed models in medical education when compared with other teaching modalities, thus it adds valuable information to the existing literature.

Second, although simple and complex CHD cases were presented to the participants in previous studies, this study included a range of cardiac diseases covering simple and complex CHD pathologies as indicated in the methods. The survey findings of these medical 

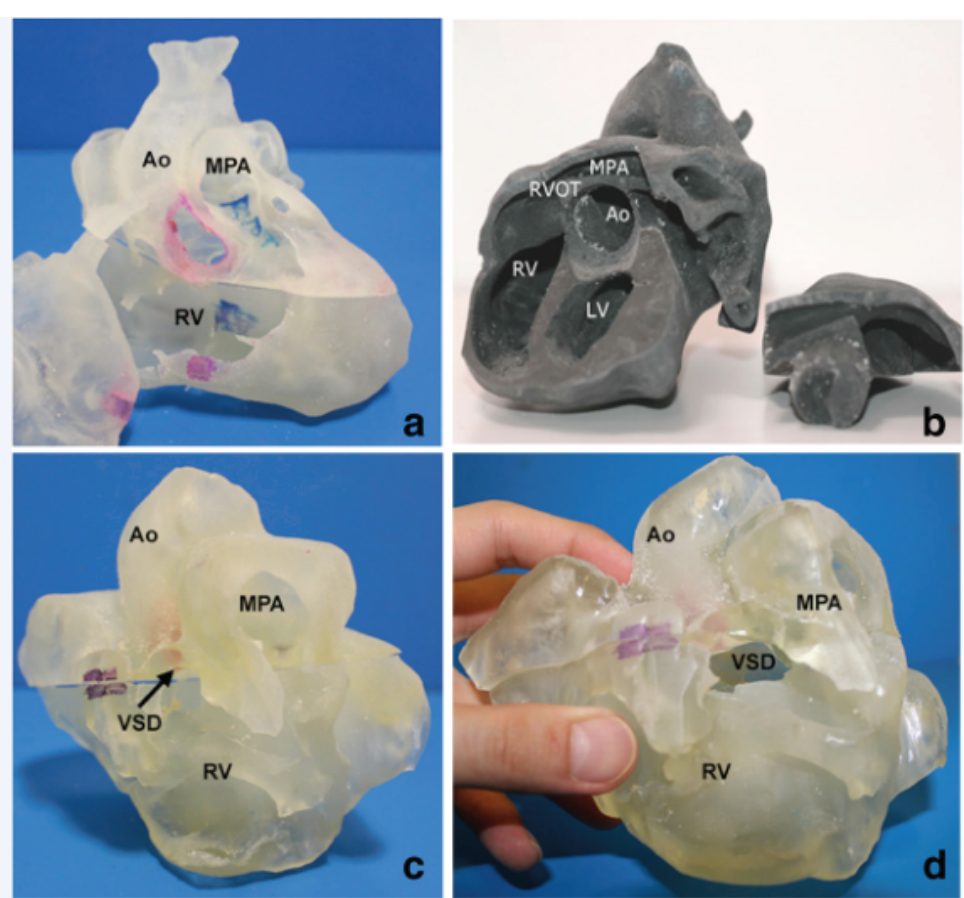

Ao - Aorta, MPA - Main Pulmonary Artery, LV - Left Ventricle, RV - Right Ventricle, RVOT - Right Ventricular Outflow Tract, VSD - Ventricular Septal Defect

Figure 1: 3D printed heart models of normal anatomy and congenital heart disease. (a) Normal heart: The model which was created from cardiac CT is partitioned into 3 pieces, including an anterior portion (the right ventricular free wall) that can be removed to visualize the normal interventricular septum. The remaining superior and inferior portions can be separated to allow for visualization of the aorta and its position relative to the right ventricle. (b) Repaired Tetralogy of Fallot (ToF) heart from an adult: The model, created from cardiac MRI is separated into 2 pieces. The cut in the main body allows for clear visualization of the pulmonary infundibular stenosis and overriding aorta. (c) Unrepaired ToF heart from an infant: The 3D model, created from 3D echocardiogram, was partitioned into 2 pieces; a superior and inferior portion divided along the Ventricular Septal Defect (VSD). (d) Unrepaired ToF heart from an infant: Separating superior and inferior portions allows for clear visualization of the VSD as well as the aortic override relative to the VSD. Reprinted with permission under the open access from Loke YH, et al [14].
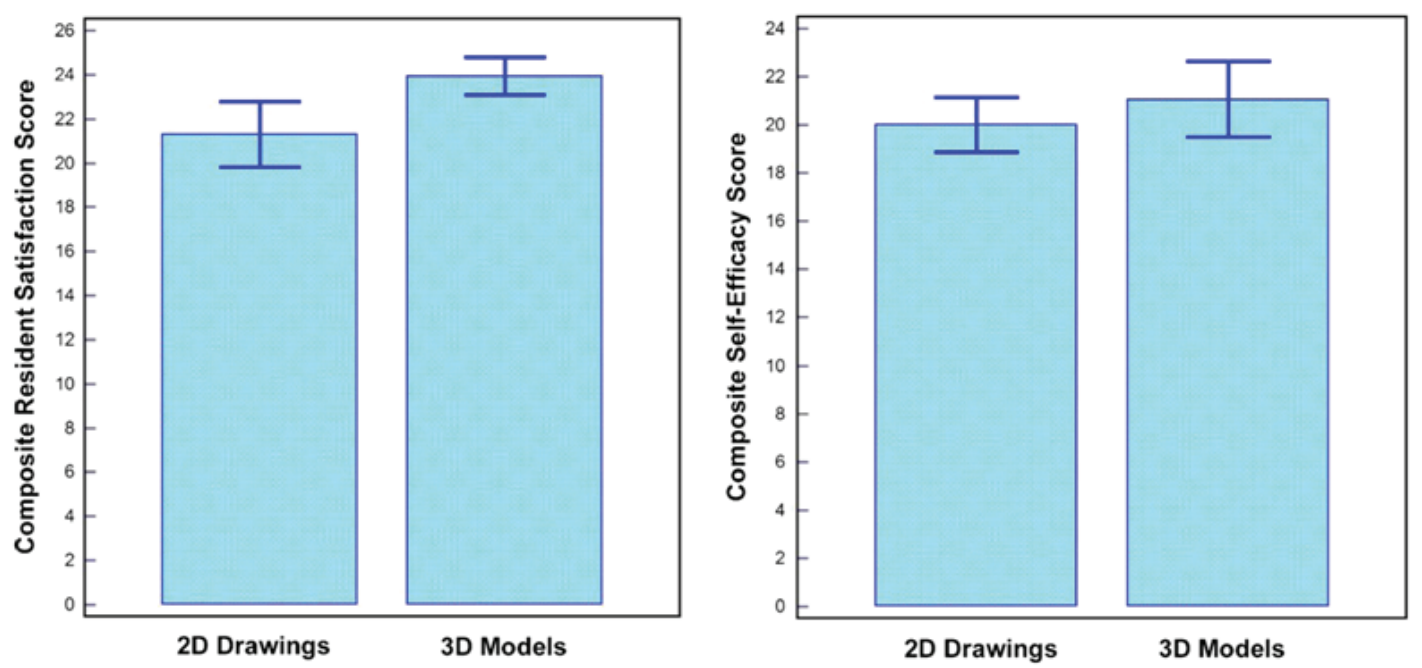

Figure 2: Impact of 3D printed heart models on medical education. Both 2D image and physical 3D model groups show improvement in knowledge based on multiple choice testing. There was a statistically significant difference in satisfaction ratings when 3D models were used. While residents in 3D model groups had higher self-efficacy aggregate scores, this difference was not statistically significant. Reprinted with permission under the open access from Loke $\mathrm{YH}$, et al [14]. 
students confirmed the significant increase in knowledge for all of the 6 CHD lesions, highlighting the usefulness of 3D printing in medical education. Furthermore, this study assessed both participants' attitudes and self-reflection regarding knowledge increase when $3 \mathrm{D}$ printed models were used as a teaching tool for various types of CHD lesions, with significant correlation noticed between knowledge increase and severity of CHD. This represents a unique aspect which has not been addressed by previous reports [13-15]. These results support the use of $3 \mathrm{D}$ printing as an effective tool in teaching $\mathrm{CHD}$, especially in the presence of complex situations.

Third, despite promising results arising from this study, it has some limitations. In addition to the small sample size as emphasized in the discussion, use of 2D diagram at one of the stations is another limitation. Given the complexity of cardiac anatomy and heterogeneous conditions associated with $\mathrm{CHD}, 3 \mathrm{D}$ image visualizations form part of the imaging tools in the diagnostic assessment of cardiovascular disease including CHD [18-20]. 3D reconstructions including volume rendering and virtual reality or augmented reality are increasingly used in clinical practice $[21,22]$, thus, this could be considered in future studies in comparison with 3D printed models.

In summary, patient-specific 3D printed models are showing great promise in medical applications especially in the field of medical student education for congenital heart disease. Current research findings provide strong evidence of increasing student's knowledge and enhancing their understanding of complex congenital heart disease with aid of 3D printed models. The benefits of 3D printed models for medical education could be classified by different types of diseases with significant benefits achieved in more complex congenital heart diseases. 3D printed realistic models are suggested to be included in the medical education program as an accurate and valuable alternative to current teaching methods.

\section{References}

1. Zanetti EM, Aldieri A, Terzini M, Cali M, Franceschini G, et al. (2017) Additively manufactured custom load-bearing implantable devices: Grounds for caution. Australasian Med J 10: 694-700.

2. Speranza D, Citro D, Padula F, Motyl B, Marcolin F, et al. (2017) Additive manufacturing techniques for the reconstruction of $3 D$ fetal faces. Appl Bionics Biomech 2017: 9701762.

3. Sun Z, Liu D (2018) A systematic review of clinical value of threedimensional printing in renal disease. Quant Imaging Med Surg 8: 311-325.

4. Perica ER, Sun Z (2018) A systematic review of three-dimensional printing in liver disease. J Digit Imaging 31: 692-701.

5. Perica E, Sun Z (2017) Patient-specific three-dimensional printing for pre-surgical planning in hepatocellular carcinoma treatment. Quant Imaging Med Surg 7: 668-677.

6. Lau I, Squelch A, Wan YL, Wong AM, Ducke W, et al. (2017) Patient-specific 3D printed model in delineating brain glioma and surrounding structures in a paediatric patient. Digit Med 3: 86-92.

7. Giannopoulos AA, Steigner ML, George E, Barile M, Hunsaker AR, et al. (2016) Cardiothoracic applications of 3-dimensional printing. J Thorac Imaging 31: 253-272.
8. Ryan J, Plasencia J, Richardson R, Velez D, Nigro JJ, et al. (2018) 3D printing for congenital heart disease: A single site's initial three-year experience. 3D Print Med 4: 10.

9. Lau IWW, Liu D, Xu L, Fan Z, Sun Z (2018) Clinical value of patientspecific three-dimensional printing of congenital heart disease: Quantitative and qualitative assessments. PLoS One 13: e0194333.

10. Sun Z, Lau I, Wong YH, Yeong CH (2019) Personalized threedimensional printed models in congenital heart disease. J Clin Med 8: E522.

11. Sun Z, Lee S (2017) A systematic review of 3D printing in cardiovascular and cerebrovascular diseases. Anatol J Cardiol 17: 423-435.

12. Lau IWW, Sun Z (2019) Dimensional accuracy and clinical value of 3D printed models in congenital heart disease: A systematic review and meta-analysis. J Clin Med 8: E1483.

13. White SC, Sedler J, Jones TW, Seckeler M (2018) Utility of threedimensional models in resident education on simple and complex intracardiac congenital heart defects. Congenit Heart Dis 13: 10451049.

14. Loke YH, Harahsheh AS, Krieger A, Olivier LJ (2017) Usage of 3D models of tetralogy of Fallot for medical education: impact on learning congenital heart disease. BMC Med Educ 17: 54.

15. Su W, Xiao Y, He S, Huang P, Deng X (2018) Three-dimensional printing models in congenital heart disease education for medical students: a controlled comparative study. BMC Med Educ 18: 178.

16. Smerling J, Marboe CC, Lefkowitch JH, Pavilicova M, Bacha E, et al. (2019) Utility of 3D printed cardiac models for medical student education in congenital heart disease: across a spectrum of disease severity. Pediatr Cardiol 40: 1258-1265.

17. Lim KH, Loo ZY, Goldie S, Adams J, McMenamin P (2016) Use of 3D printed models in medical education: A randomized control trial comparing 3D prints versus cadaveric materials for learning external cardiac anatomy. Anat Sci Educ 9: 213-221.

18. Sun Z, Squelch A, Bartlett A, Cunningham K, Lawrence-Brown M (2009) 3D stereoscopic visualization of fenestrated stent grafts. Cardiovasc Intervent Radiol 32: 1053-1058.

19. Sun Z (2011) Multislice computed tomography angiography in the diagnosis of cardiovascular disease: 3D visualizations. Front Med 5: 254-270.

20. Xu L, Sun Z (2015) Virtual intravascular endoscopy visualization of calcified coronary plaques: A novel approach of identifying plaque features for more accurate assessment of coronary lumen stenosis. Medicine (Baltimore) 94: e805.

21. Karmonik C, Elias SN, Zhang JY, Diaz O, Klucznik RP, et al. (2018) Augmented reality with virtual cerebral aneurysms: A feasibility study. World Neurosurg 119: e617-e622.

22. Witowski J, Darocha S, Kownacki L, Pietrasik A, Pietura R, et al. (2019) Augmented reality and three-dimensional printing in percutaneous interventions on pulmonary arteries. Quant Imaging Med Surg 9: 23-29. 